\title{
DISCURSO DEL RECTOR DE LA UNIVERSIDAD CATÓLICA DEL NORTE
}

\author{
Juan Music Tomicic (iD)
}

Esta es una fecha de especial significación para la Universidad Católica del Norte, y particularmente para la Sede Coquimbo, porque finalizamos hoy un ambicioso proyecto de concentrar, en el Campus Guayacán, las actividades universitarias de la Universidad en la IV Región.

Deseo agradecer muy sinceramente la presencia de las autoridades, académicos, alumnos y de todos ustedes en esta ceremonia en que se inaugura el edificio institucional de la Escuela de Derecho, cinco salas audiovisuales y un hermoseamiento urbanístico del Campus Guayacán.

Por cierto, las edificaciones no valen ni tienen un sentido por sí mismas, sino en función de los principios, misión y objetivos institucionales del proyecto académico que la sustenta y de las personas que con su aporte, sus sueños y su esfuerzo han sabido trazar la historia que desemboca en ellas y, también, en función de todos aquellos que, de diversos modos, tendrán la oportunidad de abrirse desde su seno a un futuro pleno de desafíos y proyectos.

Es en esta perspectiva donde debemos buscar el significado de lo que hoy celebramos: en la historia pasada que fue forjando esta nueva situación, y en la historia por vivir a partir de ella. Si en lo inmediato estas construcciones son el resultado de una trascendental decisión institucional y, en consecuencia, de una importante inversión de recursos económicos, lo que verdaderamente interesa explicitar es su fundamento: ¿Por qué esta decisión?. ¿En qué concepto de Universidad se apoya?, ¿Qué criterios la han guiado?

El Plan de Desarrollo Corporativo de la Universidad explicita claramente los principios, la misión y los objetivos estratégicos que han guiado las decisiones que hemos tomado en los últimos años y es en él, y en la Constitución Apostólica Ex corde Ecclesiae donde debemos buscar la respuesta a las preguntas antes formuladas. Regional.

La Universidad Católica del Norte es una Universidad Católica, Tradicional y

Como Universidad Católica nuestro quehacer se inserta en la misión amplia y evangelizadora de la Iglesia, asumiendo, entre otros aspectos, su responsabilidad en el diálogo entre el evangelio y la cultura, especialmente en el ámbito latinoamericano, y enriqueciendo su formación científica y profesional con valores éticos y humanistas que privilegian el bien común.

En toda universidad se ha de buscar aportar al perfeccionamiento del hombre y de la sociedad a través del cultivo del conocimiento, de las ciencias, en sus más diversas ramas $\mathrm{y}$, sobre todo, a través de una formación integral de nuevos profesionales para el servicio de esos mismos fines mediante su incorporación al mundo del trabajo. A la Universidad, a toda auténtica universidad, no podría bastarle, en suma, con formar 
profesionales competentes en los aspectos técnicos de una disciplina específica. Lo que se requiere más bien, es formar profesionales íntegros, fuertemente imbuidos de un humanismo y de una ética profesional y personal. Sobre esta base común a la universidad, específicamente católica, le compete el desafío de ofrecer a todos cuantos participan en ella, un proyecto verdadero y profundamente inspirado en la senda trazada por Jesucristo hacia la plenitud del hombre y de su historia.

Por lo tanto, en la medida en que una universidad católica es capaz de trascender en su visión de las cosas y en sus opciones los criterios meramente mundanos y economistas del éxito humano, y se mide más bien en el estilo de Jesús, entonces lo que ella haga se acercará más a su ideal católico. Sólo en este espíritu será posible superar aquella engañosa dicotomía entre los objetivos científico-académicos y la finalidad evangelizadora de la universidad católica, tal como lo expresa la ferviente exhortación de la Constitución Ex corde Ecclesiae: "...los estudiantes deberán ser formados en las diversas disciplinas de manera que lleguen a ser verdaderamente competentes en el campo específico al cual se dedicarán en servicio de la sociedad y de la iglesia; pero, al mismo tiempo, deberán ser preparados para dar testimonio de su fe ante el mundo".

En esta misma línea, un aspecto especialmente importante de la citada Constitución es su insistencia en la idea de que lo que ha de distinguir a una Universidad Católica no es una supuesta peculiaridad de su quehacer en el contexto general de las universidades, sino el enfoque que se dé en ella a las labores propias de la academia, como son la investigación científica, la docencia y la diversidad de servicios a la comunidad.

En otras palabras, la complejidad del proyecto fundamental de una Universidad Católica radica en el hecho de que para su auténtico desarrollo no será suficiente el despliegue de una cierta gama de actividades pastorales o de docencia teológica - por más necesarias y valiosas que éstas sean en sí mismas - puesto que el verdadero desafío es que la fe en Jesucristo y la visión del hombre y del mundo que a partir de ella ha ido decantando la Iglesia, impregnen de sentido al quehacer académico en su propia especificidad y no sean reducidas a la categoría de una dimensión meramente sobreañadida. De ahí el esfuerzo de la Constitución Ex corde Ecclesiae por explicitar los rasgos que en la universidad católica deberían caracterizar, especialmente, las labores de investigación y docencia.

Con el término Tradicional queremos expresar una Universidad que centra su quehacer no sólo en la formación de los profesionales y científicos que el país requiere sino, además, y de manera creciente en la investigación, la extensión, la formación de recursos humanos tanto a nivel de postgrado, postítulo y educación continua y en la prestación de otros servicios académicos que la sociedad requiere.

Con el término Regional queremos indicar que, sin renunciar al carácter universal de su quehacer académico, busca canalizar una parte de él a resolver los problemas y necesidades del entorno humano y geográfico en que se ubica. En este sentido si bien estamos localizados físicamente en la Segunda y Cuarta regiones del país, el término regional debemos entenderlo que abarca la subregión del Centro Oeste Sudamericano, es decir, Norte de Chile que comprende de la Primera a la Cuarta Región, y Noroeste Argentino, Bolivia y Sur del Perú. 
Estos desafíos, la universidad los aborda tomando en cuenta las realidades, necesidades, inquietudes y sueños de los habitantes del norte de Chile, los fenómenos presentes de la globalización del mundo y los "laboratorios naturales" con los que nos privilegia nuestra geografía.

En los conceptos antes planteados, y otros, que por razones de tiempo no se han explicitado, debemos buscar la respuesta a las decisiones que hemos tomado en la Universidad y particularmente en la Sede Coquimbo, estos últimos años.

Al asumir como Rector el año 1990, la Sede Coquimbo de nuestra Universidad ofrecía a los egresados de enseñanza media dos carreras: Biología Marina e Ingeniería en Acuacultura, y el universo estudiantil en la sede era de 480 alumnos. Basado en esta realidad, la Universidad toma la decisión de ampliar las ofertas educacionales en la IV Región creando las carreras de Ingenieria Comercial, Derecho, Licenciatura en Ciencias Religiosas y Pedagogía en Filosofía y Religión, creando, además, un programa de Magister en Ciencias del Mar y ampliando las ofertas al nivel de educación continua. Estas decisiones han permitido a esta Casa de Estudios Superiores dar más oportunidades a los jóvenes de esta región y de la zona norte del país, y crear cada vez nuevas oportunidades de reciclaje o actualización de conocimiento a los profesionales que ejercen en la zona. Todas estas decisiones han implicado una fuerte inversión en recursos humanos, nueva infraestructura y equipamiento docente, mayores beneficios para los estudiantes y diseño de planes de estudio innovadores y actualizados. Todo lo anterior ha implicado que el universo estudiante sea actualmente de 1.450 jóvenes, en la Sede Coquimbo.

Posteriormente a la creación de las nuevas carreras, se realizó un completo estudio que significó tomar dos decisiones importantes:

Concentrar todas las actividades universitarias de la Sede Coquimbo, en el Campus Guayacán,

Crear en el Campus Miraflores un Centro de Formación de Técnicos, administrado a través de una Fundación, creada por la propia Universidad.

La decisión de concentrar las actividades universitarias de la Sede en el Campus Guayacán, implicó la elaboración de un plano regulador del Campus que definiera claramente la zonificación de él y la ubicación que tendrían las futuras dependencias que se construyeran, de acuerdo a las necesidades académicas de las carreras antes mencionadas y de nuevas áreas de conocimiento que creara, a futuro, la institución.

Cuando se visualiza una idea y se proyecta el desarrollo de una institución a futuro existen pensamientos firmes sobre el particular pero, a su vez, inquietudes sobre la real factibilidad de materializar estos sueños planificados. Cuando en 1992, y aún años antes, se intentaba proyectar esta Sede en la IV Región para desarrollarla con nuevas áreas de conocimiento fluía la idea de la creación de una Escuela de Derecho lo que constituía, indudablemente, un gran desafío porque nuestra Universidad nunca habia incursionado en esta disciplina.

Sin embargo, tuvimos la suerte de encontrar valiosos profesionales colaboradores que, con su preparación y entusiasmo, nos ayudaron en lo que finalmente constituyó el "Proyecto Escuela de Derecho, Universidad Católica del Norte, Sede Coquimbo". Es así como finalmente y después de la aprobación del $\mathrm{H}$. Consejo Superior de la Uni- 
versidad, nace la Escuela y la Carrera de Derecho, en marzo de 1993, en el Campus Miraflores de nuestra Universidad.

Los años fueron transcurriendo, la carrera progresando año tras año, incrementándose el número de alumnos, profesores, libros, etc., en un recinto que se hacía estrecho y por qué no decirlo, no contaba con las comodidades y facilidades adecuadas. Simultáneamente otras actividades académicas de la Universidad se desarrollaban en el mismo Campus Miraflores y ellas también sufrían similares limitaciones de infraestructura, antecedentes que se tuvieron en cuenta para ir gestando la idea central que hoy vemos realizada, esto es, concentrar todas las actividades académicas que desarrolla nuestra Universidad en la IV Región en el Campus Guayacán.

Fue una tarea larga, compleja y bastante onerosa para nuestra Universidad, pero poco a poco el proyecto matriz se fue coronando y es así como en 1994 se traslada a Guayacán la carrera de Ingeniería Comercial que había sido creada en 1993, la que finalmente encuentra su lugar definitivo en el edificio que se construye especialmente para ella y que se inauguró en el mes de octubre de 1996.

El Departamento de Teología, creado en el año 1974, funcionó por 24 años en el Campus Miraflores, y se traslada finalmente, con las dos carreras que sustenta, al Campus Guayacán al inaugurarse su actual edificio institucional el 20 de marzo de 1998.

Se va preparando el Campus Guayacán para recibir a los nuevos académicos y alumnos en las mejores condiciones posibles, es así entonces como simultáneamente se construyen en este Campus nuevas salas de clases, completo sistema sanitario, se remodela la Biblioteca, se implementa la Red Internet, y se construye y se entrega a la comunidad, el pasado mes de agosto, nuestro Casino Estudiantil que nos enorgullece.

Junto con todo lo anterior, y para coronar este esfuerzo, la Universidad acaba de inaugurar ayer una Sala de Lectura que, como ampliación de Biblioteca, permitirá satisfacer todas las necesidades de nuestros alumnos y académicos. $Y$ hoy en día, con especial emoción, estamos inaugurando y entregando a la comunidad este magnífico edificio para la Escuela de Derecho, junto con cinco salas de clases.

Estas dependencias se han diseñado pensando en todos los requerimientos de una moderna Escuela de Derecho que deberá enfrentar los desafíos que implica la modernización del sistema jurídico chileno.

Las obras que hoy inauguramos significaron la construcción de $1.293 \mathrm{~m} 2$ de nuevos edificios, el hermoseamiento del Campus, más zona de estacionamiento. El costo total de la inversión, considerando edificios más equipamiento, fue de 577 millones de pesos.

Esta concentración de todas las actividades académicas en nuestro Campus Guayacán, permitirá a la Universidad una administración más eficiente y, lo que es más importante, una valiosa integración de todos sus alumnos y funcionarios y, por lo tanto, un enriquecimiento de la vida académica al interactuar disciplinas, a primera vista, tan diversas pero que en el complejo mundo en que vivimos se integran entre sí en la multidisciplinariedad con que actualmente deben enfrentarse los complejos problemas que tiene nuestra sociedad. Nosotros esperamos que esta integración sea intensa, favorecida por la concentración física pero, más aún, impulsada por los propios académicos y 
estudiantes que deben entender que los antiguos feudos disciplinarios se abren en todo el mundo para enriquecerse mutuamente.

Nada de esto hubiera sido posible sin la comprensión de profesores y estudiantes de la Escuela de Derecho quienes, poco a poco, fueron integrándose a la idea y ayudando a completarla. Conocemos las diferentes aspiraciones de los integrantes de esta Escuela y agradecemos a todos, muy profundamente, el apoyo que directa o indirectamente dieron a esta iniciativa, y esperamos que toda la comunidad de esta Escuela se sienta cómoda, contenta y motivada en este nuevo lugar de trabajo.

Quisiera agradecer muy especialmente a la fundadora y Directora de la Escuela señora Luz María Reyes Santelices, sus desvelos y dedicación para conseguir el exitoso desarrollo académico de ella, ya que la infraestructura no tiene importancia si no existen de por medio las personas comprometidas con un proyecto. La Directora y los diferentes profesores han colaborado desde un principio en esta carrera con tal seriedad y dedicación que estamos orgullosos por la calidad profesional que se ha logrado en los Abogados que estamos formando.

Ahora, si a la calidad le agregamos este nuevo edificio con toda la infraestructura necesaria, naturalmente nos sentimos orgullosos porque a lo humano se agrega lo físico formando un complemento que sin lugar a dudas nos asegurará mantener la calidad de esta Escuela como una de las mejores de Chile.

Muchas personas, instituciones, y organizaciones distintas, junto a personas particulares nos apoyaron, desde los inicios, en la creación de la carrera de Derecho. En esta oportunidad yo quisiera resaltar en las personas aquí presentes, ese apoyo que fue fundamental para la creación y continuación de la carrera: primeramente al señor Intendente de la Cuarta Región Coquimbo, don Renán Fuentealba Moena; al señor Rector de la Pontificia Universidad Católica de Chile don Juan de Dios Vial Correa, quien junto al Vicedecano de la Facultad de Derecho de ese entonces, hoy Profesor Titular de Derecho Penal, Criminología y Psicología Jurídica de la misma Universidad, don Jaime Náquira Riveros, apoyaron a esta Universidad desde antes de la creación de la carrera, a través de un convenio de cooperación y posteriormente en un protocolo, que fueron suscritos entre ambas instituciones y que permitió el exitoso desarrollo del programa; al Colegio Regional de Abogados A.G. de la IV Región; a la llustrísima Corte de Apelaciones de La Serena; a la Asociación de Mujeres Abogados de la IV Región, y a cada uno de los muchos prestigiosos Abogados que de alguna forma han cooperado para lograr el éxito de la carrera. Todo este bagaje de apoyo y experiencia nos permitirá que cuando nuestra carrera se someta al sistema de acreditación que impulsa el Ministerio de Educación, salga exitosamente evaluada.

Gracias a cada uno de los colaboradores aludidos, y de los que se nos pueda olvidar mencionar en este acto.

Para toda nuestra comunidad universitaria el haber dado término al proyecto de concentrar en este Campus todas las actividades académicas de la Universidad es una gran satisfacción pero, en el largo plazo no representa más que una etapa más en el devenir de la institución. Debemos visualizar las nuevas etapas que como gran desafio enfrentaremos a futuro. La primera es indudablemente consolidar la enseñanza de pregrado de nuestras seis carreras, mejorando la docencia en todos sus aspectos. Luego debemos incrementar los proyectos de investigación que realizan nuestros académicos, 
porque así fortaleceremos nuestro aporte a la ciencia y al mejoramiento de la docencia. A su vez, y en forma simultánea, debemos ampliar y profundizar las actividades de extensión en toda la Cuarta Región en torno a las disciplinas que cultivamos y a los problemas regionales de interés.

Pero el desafío de cualquiera institución universitaria es más amplio aún, porque debe estar siempre visualizando el futuro e identificando nuevas necesidades que satisfacer. Para este Rector es de suma importancia precisar ante ustedes y ante la comunidad toda, que nuestra Universidad está avanzando minuciosamente en los diversos aspectos técnicos del estudio sobre la creación de la futura carrera de medicina. Precisamente, y es una gran coincidencia que, en la misma forma como hace ya siete años, firmábamos el convenio de cooperación con la Pontificia Universidad Católica de Chile para la creación de la carrera de Derecho, hace algunos meses, hemos firmado otro convenio con esta Casa de Estudios Universitarios, a través del cual su prestigiosa Facultad de Medicina nos dará apoyo académico para el proyecto que en estos momentos estamos impulsando. Vaya para esta Casa de Estudios nuestro más preciado agradecimiento como también a todos los médicos de la zona, a nuestro Arzobispo y Representante del Gran Canciller de nuestra Universidad Monseñor Manuel Donoso Donoso, a representantes de servicios públicos y a nuestros académicos que están involucrados en la elaboración de la futura Escuela de Medicina de la IV Región.

De esta manera creemos que estamos dando cabal cumplimiento a los compromisos que, históricamente, la Universidad se planteó cuando las autoridades de aquel tiempo decidieron crear esta Sede en 1968 y que en estos días cumplirá por lo tanto 31 años de presencia en esta IV Región.

Pero nuestro compromiso con la comunidad de esta región quedaría inconcluso si no mencionara lo que, en buena medida, dio origen al establecimiento de nuestra Universidad en ella, cuando el entonces Arzobispo de La Serena Monseñor Francisco Fresno Larraín solicitó, en el año 1967, la presencia de nuestra Universidad, y lo hizo pensando especialmente en carreras técnicas y de pedagogía para apoyar el desarrollo, en especial, de la entonces Provincia de Coquimbo que pasaba por críticos momentos económicos. Ahora con satisfacción podemos informar de la creación de un Centro de Formación Técnica que, dependiendo de una Fundación creada por nuestra Universidad, empieza a funcionar a partir de este mes de marzo, en lo que fue el origen de la propia Universidad: el Campus Miraflores, a la salida norte de Coquimbo. Ese Centro que ofrecerá inicialmente seis carreras técnicas de nivel superior satisfará, en parte, el desarrollo que todos esperamos de esta región.

Así la Universidad Católica del Norte desarrolla su quehacer intentando no descuidar ningún segmento de la sociedad pero, enfatizando en lo que le es más propio en su calidad de Centro de Estudios Superiores: la Educación Superior.

Permítanme recordar que para finalizar el proyecto que hoy inauguramos, la Universidad ejecutó un ambicioso plan de inversiones en el Campus Guayacán que significó:

- Construcción de una nueva biblioteca.

- Construcción y remodelación del Auditorium.

- Construcción del Laboratorio de Computación. 
- Construcción de 4 salas de clases audiovisuales de 100 alumnos cada una.

- Construcción del Centro de Producción de Abalón.

- Ampliación, remodelación y mejoramiento del sector estudiantil.

- Construcción del edificio de Ingeniería Comercial.

- Construcción del edificio del Departamento de Teología.

- Construcción de un nuevo Casino con capacidad para 300 alumnos.

- Pavimentación del campus y creación de zonas de estacionamiento.

- Construcción del Laboratorio de Hidráulica.

- Construcción de la Sala de Lectura y Estudios para cobijar a 150 alumnos, y que inauguramos en el día de ayer, y

Las obras que hoy inauguramos del edificio institucional de la Escuela de Derecho y cinco salas de clase audiovisuales.

Todo este plan significó - en moneda de hoy - una inversion total en infraestructura física y equipamiento de 2.633 miliones de pesos. A lo anterior debe agregarse las inversiones que se han realizado en la contratación de nuevo personal académico y no académico, para la Sede.

Como puede verse, ha sido política de la Universidad ser muy cuidadosa cuando se crean nuevas carreras en el sentido de considerar, además de un plan de estudio y programas innovadores y actualizados, las inversiones que se deben realizar tanto en recursos humanos como en infraestructura física y de equipamiento que ellas requieren. Todo lo antes señalado avalan el fiel cumplimiento que hemos tenido con lo comprometido. La seriedad, responsabilidad y calidad de todo nuestro quehacer académico es nuestro norte y compromiso con todos ustedes y la región a la que nos debemos. Pueden estar seguros que la Universidad seguirá con esta política a futuro.

No puedo finalizar esta intervención sin antes expresar mi profundo reconocimiento y agradecimiento a diferentes instancias e instituciones que colaboraron en la materialización de este proyecto. En primer término agradecer al Honorable Consejo Superior de la Universidad el apoyo y aprobación al plan de inversiones del que esta construcción forma parte.

Al Ministerio de Educación que, a través del Fondo Concursable de Desarrollo Institucional, financió 109 millones de pesos para la materialización de esta obra.

A todos los directivos de la Sede que, durante estos años, impulsaron y colaboraron en la materialización de este proyecto, en especial a los ex - Vicerrectores de Sede don Exequiel González y don Mario Edding, al ex - Secretario de Sede don Alfonso Silva y a los actuales directivos don Renzo Follegati y don Misael Camus. A la Directora de la Escuela de Derecho, sus académicos, alumnos y personal administrativo, por su comprensión y dedicación en especificar los requerimientos que tenía que cumplir este proyecto.

A la empresa de la región "Arquitectura y Construcción Rencoret Ltda,", que tuvo la delicada misión de la construcción de la obra. 
Al arquitecto de la Universidad don Sergio Gaete Thomas, que proyectó esta obra, y a don Juan Carlos Cárcamo quien no sólo controló su adecuada materialización sino, se preocupó de que cada detalle y equipamiento correspondiera a lo solicitado por la Escuela de Derecho.

A todo el personal de la Sede que facilitó la labor constructiva y resolvió ágilmente cualquier problema presentado.

Es para mi un verdadero privilegio entregar en el día de hoy, oficialmente, esta obra y agradecer muy sinceramente la presencia de todos ustedes en este acto.

Sigamos trabajando unidos para engrandecer cada día más esta Sede y por lo tanto nuestra querida Universidad. 